\title{
Stress-strain assessment of plain dents in gas pipelines
}

\author{
Yaroslav Dubyk \\ IPP-Centre LLC, Ukraine \\ dubykir@gmail.com, bttp:/ / orcid.org/0000-0002-4327-412X
}

\section{Iryna Seliverstova}

G. S. Pysarenko Institute for Problem of Strength, National Academy of Sciences of Ukraine, Ukraine

seliverstovairyna24@gmail.com, bttp:/ / orcid.org/0000-0003-1166-792X

\begin{abstract}
This paper presents the analytical solution of the stress-strain state for a dented pipeline, based on the method of equivalent loads. Firstly, a solution for a harmonic imperfection was found, then using Fourier series expansion a semi-analytical procedure was proposed to assess a single dent. A comparison between analytical and numerical results for the axial force and pressure load were given. The influence of the dent dimensions, shell radius to thickness ratio and initial loading to stress concentration factor were discussed.
\end{abstract}

KEYWORDS. Cylindrical shell; Plane dent; Approximate solution;

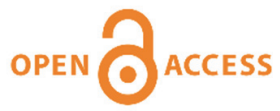

Citation: Dubyk Y., Seliverstova I., Stressstrain assessment of plain dents in gas pipelines, Frattura ed Integrità Strutturale, 51 (2020) 459-466.

Received: 11.11 .2019

Accepted: 06.12.2019

Published: 01.01.2020

Copyright: (C) 2020 This is an open access article under the terms of the CC-BY 4.0, which permits unrestricted use, distribution, and reproduction in any medium, provided the original author and source are credited.

\section{INTRODUCTION}

runk pipelines are one of the most common means used in the transportation of various kinds of energy resources. Ensuring trouble-free operation of pipelines is a pledge of both the environmental and energy security of the country through which they pass. The most common cause of accidents on trunk pipelines is the presence of mechanical damage (dents and gauges) and corrosion [1]. Therefore, the key point in the study of the technical condition and the extension of the life of trunk pipelines is the determination of their stress state, taking into account these local damages. Dents are one of the most common types of defects arising due to external interaction - damage by heavy machinery (excavators, tractors, pipe layers), falling stones on top of a pipeline or resting on supports [2].

Local imperfections like dents on pipelines are quite complicated for engineering analysis. To assess this problem, contact mechanics and nonlinearities in both material and geometry have to be considered in all phases of the dent life cycle.

Recently these problems are addressed using FEA only [3, 4]. Finite Element method is the most largely used method for solving problems of engineering but since it is a numerical study it does not give the total accurate understanding of the problem. To investigate dent problems, it would be best to develop a closed-form analytical model would be the wisest choice to investigate dent problems but it is almost impossible due to the complicated geometry and it doesn't give considerable advantages comparing to FEM analysis. Instead a semi-analytical numerical procedure is proposed in the current study based on the equivalent load method. 
Equivalent loads method was proposed by [5], to study the stress behavior of thin-shell structures containing geometric imperfections. The stress behavior of a geometrically imperfect shell under load is equivalent to the sum of two stress fields [6]. The first stress field is produced by the original load acting on the geometrically perfect version of the shell. The second stress field results from applying to the perfect version of the shell a load pattern that results in perturbation stress behavior equivalent to that which would be induced by the geometric imperfection. It should be noted that the equivalent load does not produce the correct dent shape but instead produce a stress field that is similar to that which would have been produced by the dent. Equivalent load is given usually in the form [6]:

$$
p \approx N_{x} \chi_{x x}+2 N_{x \varphi} \chi_{x \varphi}+N_{\varphi \varphi} \chi_{\varphi \varphi}
$$

$N_{x}, N_{x \varphi}, N_{\varphi \varphi}-$ membrane forces, that arise from the action of the initial load on the curved shell;

$\chi_{x}, \chi_{\varphi \varphi}, \chi_{x \varphi}$ - change curves in two main directions and the change curvature in twist.

Eq. (1) is standard in the solution of non-ideal shell problems and is used by [7], who proposed to improve its accuracy by considering the additional terms for imperfections amplitudes larger than the shell thickness [8].

Modern regulatory documents [9] in the damage risk analysis consider only the permissible depth of such defects, neglecting other geometrical parameters and the load level. The main idea of a current study is to develop effective semi-analytical procedure for dent analysis based on equivalent loads method with second order terms to perform the convergency analysis and to investigate the dent shape effect and loading level on the stress concentrator factor.

\section{ANALYTICAL BACKGROUND}

\section{General equations}

7 he theory of the thin-shells is quite well-known and effective for pipelines application. General equation of cylindrical shell equilibrium under external forces:

$$
[\mathbf{L}]\{\mathbf{u}\}=\{\mathbf{F}\}
$$

$\{\mathbf{u}\}=[u v w]^{T}$ is the displacement vector;

$u, v$ and $w$ are the orthogonal components of displacements in the $x, \varphi$ and radial directions;

$\{\mathbf{F}\}=R^{2} / H\left[\begin{array}{lll}p_{x} & p_{\varphi} & p_{r}\end{array}\right]^{T}$ is the external loading vector;

$[\mathbf{L}]$ is a matrix differential operator [10]:

$$
[\mathbf{L}]=\left[\mathbf{L}_{D-M}\right]+k\left[\mathbf{L}_{M O D}\right]+\frac{1}{H}\left[\mathbf{L}_{I N I}\right]
$$

Differential matrix operator $\left[\mathbf{L}_{D-M}\right]$-corresponds to Donnel-Mushtari shell theory, the simplest one;

$\left[\mathbf{L}_{M O D}\right]$ - modifying operator, for a different shell theory, further we will use Flugge shell theory;

$\left[\mathbf{L}_{I N I}\right]$-differential operator which implies the initial stress influence.

These operators are written in explicit form below:

$$
\left[\mathbf{L}_{D-M}\right]=\left[\begin{array}{ccc}
\frac{\partial^{2}}{\partial s^{2}}+\frac{1-\mu}{2} \frac{\partial^{2}}{\partial \varphi^{2}} & \frac{1+\mu}{2} \frac{\partial^{2}}{\partial s \partial \varphi} & \mu \frac{\partial}{\partial s} \\
\frac{1+\mu}{2} \frac{\partial^{2}}{\partial s \partial \varphi} & \frac{\partial^{2}}{\partial \varphi^{2}}+\frac{1-\mu}{2} \frac{\partial^{2}}{\partial s^{2}} & \frac{\partial}{\partial \varphi} \\
\mu \frac{\partial}{\partial s} & \frac{\partial}{\partial \varphi} & 1+k \nabla^{4}
\end{array}\right]
$$




$$
\left[\mathbf{L}_{M O D}\right]=\left[\begin{array}{ccc}
\frac{1-\mu}{2} \frac{\partial^{2}}{\partial \varphi^{2}} & 0 & -\frac{\partial^{3}}{\partial s^{3}}+\frac{1-\mu}{2} \frac{\partial^{3}}{\partial s \partial \varphi^{2}} \\
0 & 3 \frac{1-\mu}{2} \frac{\partial^{2}}{\partial s^{2}} & -3 \frac{1-\mu}{2} \frac{\partial^{3}}{\partial^{2} s \partial \varphi} \\
-\frac{\partial^{3}}{\partial s^{3}}+\frac{1-\mu}{2} \frac{\partial^{3}}{\partial s \partial \varphi^{2}} & -3 \frac{1-\mu}{2} \frac{\partial^{3}}{\partial^{2} s \partial \varphi} & 1+2 \frac{\partial^{2}}{\partial \varphi^{2}}
\end{array}\right]
$$

$$
\left[\mathbf{L}_{I N I}\right]=\left[\begin{array}{ccc}
N_{\varphi} \frac{\partial^{2}}{\partial \varphi^{2}}+N_{x} \frac{\partial^{2}}{\partial s^{2}}+2 N_{x \varphi} \frac{\partial^{2}}{\partial x \partial \varphi} & 0 & -N_{\varphi} \frac{\partial}{\partial s} \\
0 & N_{\varphi} \frac{\partial^{2}}{\partial \varphi^{2}}+N_{x} \frac{\partial^{2}}{\partial s^{2}}+2 N_{x \varphi} \frac{\partial^{2}}{\partial x \partial \varphi} & -3 \frac{1-\mu}{2} \frac{\partial^{3}}{\partial^{2} s \partial \varphi} \\
-N_{\varphi} \frac{\partial}{\partial s} & N_{\varphi} \frac{\partial}{\partial \varphi}+2 N_{x \varphi} \frac{\partial}{\partial x} & -N_{\varphi} \frac{\partial^{2}}{\partial \varphi^{2}}-N_{x} \frac{\partial^{2}}{\partial s^{2}}-2 N_{x \varphi} \frac{\partial^{2}}{\partial x \partial \varphi}
\end{array}\right]
$$

The following notations are used:

$$
k=\frac{h^{2}}{12 R^{2}}=\frac{\delta}{R^{2}} ; H=\frac{E h}{1-\mu^{2}} ; D=H \delta ;
$$

$\mathrm{R}$ - shell radius,

$b-$ shell thickness,

E-Young's modulus,

$\mu-$ Poisson ratio.

The complete explicit solution of Eq. (2) can be found only for simplest geometries, and loadings, for a single dent it can't be obtained, thus a numerical procedure is developed below based on the accurate solution for the harmonic imperfection and Fourier series expansion.

\section{Harmonic imperfection}

A harmonic imperfection was considered as a base for further solution and the displacements representation can be found using:

$$
u=C_{m n}^{u} \cos (n \varphi) \sin \left(\frac{\lambda}{R} x\right), v=C_{m n}^{v} \sin (n \varphi) \cos \left(\frac{\lambda}{R} x\right), w=C_{m n}^{w} \cos (n \varphi) \cos \left(\frac{\lambda}{R} x\right)
$$

$\lambda=m \pi \mathrm{R} / l$,

$n, m-$ wave number in circumferential and axial directions,

$l$ - length of the shell,

$C_{m n n}^{n}, C_{m n}^{v}, C_{m n}^{w}$ - modes coefficients for corresponded directions.

Substituting representations (8) in Eq. (2), we can get a simple algebraic set of equations:

$$
\begin{aligned}
& C_{m n n}^{n} \frac{1}{2}\left(\begin{array}{l}
k \mu n^{2}-k n^{2}+n^{2} \mu-2 \lambda^{2} \\
-n^{2}-2 \frac{N_{x} \lambda^{2}+N_{\varphi} n^{2}}{H}
\end{array}\right)+C_{m n n}^{v} \frac{\lambda n}{2}(1+\mu)-C_{m n n}^{w} \frac{\lambda}{2}\left(k \mu n^{2}+2 k \lambda^{2}-k n^{2}+2 \mu-2 \frac{N_{\varphi}}{H}\right)=0 \\
& -C_{m n}^{u} \frac{\lambda n}{2}(1+\mu)+C_{m n}^{v} \frac{1}{2}\left(\begin{array}{l}
3 k \mu \lambda^{2}-3 k \lambda^{2}+\lambda^{2} \mu-2 n^{2}- \\
\lambda^{2}-2 \frac{N_{x} \lambda^{2}+N_{\varphi} n^{2}}{H}
\end{array}\right)-C_{m n}^{w} \frac{n}{2}\left(k \mu \lambda^{2}-3 k \lambda^{2}-2-2 \frac{N_{\varphi}}{H}\right)=0
\end{aligned}
$$




$$
\begin{aligned}
& C_{m n}^{u} \frac{\lambda}{2}\left(k \mu n^{2}+2 k \lambda^{2}-k n^{2}+2 \mu-2 \frac{N_{\varphi}}{H}\right)-C_{m n}^{v} \frac{n}{2}\left(k \mu \lambda^{2}-3 k \lambda^{2}-2-2 \frac{N_{\varphi}}{H}\right)+ \\
& +C_{m n}^{w}\left(k \lambda^{4}+2 k \lambda^{2} n^{2}+k n^{4}-2 k n^{2}+k+1+2 \frac{N_{x} \lambda^{2}+N_{\varphi} n^{2}}{H}\right)=N_{\varphi \varphi} \xi \frac{n^{2} R l}{m \pi}+N_{x x} \xi \frac{n^{2} R \pi m}{l}
\end{aligned}
$$

This set of equations can be easily solved using any mathematical software, like Maple or Mathematica, but its solution is too long to be given here. The solution of a harmonic imperfection is itself useful, as it can be used to approximate the long dent, but the solution for a single dent can be further obtained using Fourier series expansion.

\section{Solution for a single dent}

Dents can have different profiles, which depend on the mechanism of dent formation and working conditions. To work with a specific dent, the profile should be measured directly and afterwards approximated by a Fourier expansion but, to test the analytical procedure, the following smooth shape function was used to describe the dent profile:

$$
R(x, \varphi)=R-\xi \exp \left(-\frac{1}{2}\left(\frac{\varphi}{\varphi_{0}}\right)^{2}\right) \exp \left(-\frac{1}{2}\left(\frac{x}{x_{0}}\right)^{2}\right)
$$

$x_{0}$ - length of the dent in the axial direction,

$\varphi_{0}$ is the length in circumferential direction,

$\xi$ - dent depth.

A very common way to deal with single imperfections is to use Fourier expansion. Thus, to find a solution for a dent, load coefficients (1) for every mode, using double integrals, have to be found:

$$
\begin{aligned}
& p_{0}=\frac{1}{\pi} \frac{1}{l} N_{x}^{*} \frac{\xi_{m, n}}{x_{0}^{2}} \int_{-\pi}^{\pi} \int_{-l}^{l}\left(\left(\frac{x}{x_{0}}\right)^{2}-1\right) \exp \left(-\frac{1}{2}\left(\frac{\varphi}{\varphi_{0}}\right)^{2}\right) \exp \left(-\frac{1}{2}\left(\frac{x}{x_{0}}\right)^{2}\right) d x d \varphi \\
& p_{x x}=\frac{1}{\pi} \frac{1}{l} N_{x}^{*} \frac{\xi_{m, n}}{x_{0}^{2}} \int_{-\pi-l}^{\pi} \int\left(\left(\frac{x}{x_{0}}\right)^{2}-1\right) \exp \left(-\frac{1}{2}\left(\frac{\varphi}{\varphi_{0}}\right)^{2}\right) \exp \left(-\frac{1}{2}\left(\frac{x}{x_{0}}\right)^{2} \cos (n \varphi) \cos \left(\frac{\lambda}{R} x\right)\right) d x d \varphi \\
& p_{\varphi \varphi}=\frac{1}{\pi} \frac{1}{l} N_{\varphi}^{*} \frac{\xi_{m, n}}{\varphi_{0}^{2}} \int_{-\pi-l}^{\pi} \int_{-l}^{l}\left(\left(\frac{\varphi}{\varphi_{0}}\right)^{2}-1\right) \exp \left(-\frac{1}{2}\left(\frac{\varphi}{\varphi_{0}}\right)^{2}\right) \exp \left(-\frac{1}{2}\left(\frac{x}{x_{0}}\right)^{2} \cos (n \varphi) \cos \left(\frac{\lambda}{R} x\right)\right) d x d \varphi
\end{aligned}
$$

These integrals can be computed numerically, so it's possible to deal with any dent form, thus, in contrast to other analytical solutions, the dent form influence can be further investigated. Also, it is very useful for analysing the dents found during the inspection. For a complete solution, internal forces and moments with a simple summing for each mode must be found:

$$
\begin{aligned}
& N_{x}=\frac{H}{R} \sum_{n} \sum_{m}\left[C_{m n}^{u} \lambda+C_{m n}^{v} \mu n+C_{m n}^{w}\left(\mu+k \lambda^{2}\right)\right] \cos n \varphi \cos \frac{x}{R} \lambda \\
& N_{\varphi}=\frac{H}{R} \sum_{n} \sum_{m}\left[C_{m n n}^{u} \lambda \mu+C_{m n}^{v} n+C_{m n}^{w}\left(1-k n^{2}+k\right)\right] \cos n \varphi \cos \frac{x}{R} \lambda \\
& M_{x}=-\frac{D}{R^{2}} \sum_{n} \sum_{m}\left[C_{m n}^{u} \lambda+C_{m n}^{v} \mu n+C_{m n n}^{w}\left(\lambda^{2}+\mu n^{2}\right)\right] \cos n \varphi \cos \frac{x}{R} \lambda
\end{aligned}
$$




$$
M_{\varphi}=-\frac{D}{R^{2}} \sum_{n} \sum_{m} C_{m n}^{w}\left(\lambda^{2} \mu+n^{2}-1\right) \cos n \varphi \cos \frac{x}{R} \lambda
$$

Stresses were obtained using the following formulas of the shell theory, and were divided into membrane and bending part, which is very common for nuclear, oil and gas industries:

$$
\begin{gathered}
\sigma_{x}=\underbrace{\frac{N_{x}}{b}}_{\sigma_{m x}} \pm \underbrace{\frac{6 M_{x}}{b^{2}}}_{\sigma_{b x}} \\
\sigma_{\varphi}=\underbrace{\frac{N_{\varphi}}{b}}_{\sigma_{m \varphi}} \pm \underbrace{\frac{6 M_{\varphi}}{b^{2}}}_{\sigma_{h \varphi}}
\end{gathered}
$$

In the following section, this semi-analytical procedure will be tested in detail, and some properties will be discussed

\section{RESULTS AND DisCUSSION}

o analyze the dent behavior, the stress concentration factor will be introduced, which is the ratio of a local stress caused by the dent to the nominal stress level in a perfect pipe:

$$
\begin{aligned}
& S C F_{\text {fore }}=\frac{\sigma_{m x x} \pm \sigma_{b x}}{\sigma_{\text {nom }}} \\
& S C F_{\text {pressure }}=\frac{\sigma_{m \varphi} \pm \sigma_{b \varphi}}{\sigma_{\text {nom }}}
\end{aligned}
$$

Here $\sigma_{n o m}$ is a nominal stress level in a shell without a dent. In this study, two main loadings for oil and gas pipelines internal pressure $(\mathrm{P})$ and axial force $(\mathrm{F})$ were considered:

$$
\begin{gathered}
\sigma_{\text {nom forre }}=\frac{N_{x}}{h}=\frac{F}{2 \pi R h} \\
\sigma_{\text {nom pressure }}=\frac{N_{\varphi}}{b}=\frac{P R}{b}
\end{gathered}
$$

First of all, the convergence criteria will be considered to the analysis, i.e. how many modes should be included in Fourier expansion. On Fig. 1, the convergence solution can be seen with respect to the number of modes involved (number of terms in Fourier expansion) and it is possible to conclude that 50th-60th modes were quite enough for dent assessment. Also, for internal pressure loading, it's possible to note that the proposed solution converges faster.

On Fig. 2 and Fig. 3, a comparison with numerical results obtained by the commercial FEA ANSYS can be seen. As an applied load axial force (see Fig. 2) and pressure (Fig. 3) were used, the nominal stress level was equal to $1 \mathrm{MPa}$, note that the stress categorization according to Eqns. (20) and (21) was used. However, an equivalent load method underestimates the peak stress concentrated value, it predicts quite well the stress profile and from Fig. $1 \mathrm{~b}$ it can be seen that 20-25 modes will be enough for profile prediction.

The influence of the nominal stress level was analyzed in Fig. 4. It can be seen the continuous reduction of the SCF with the increasing of the stress level, this effect is introduced in the model with the initial stress matrix (6), without it horizontal lines would be obtained. The initial stress influence can be explained by the fact that high level forces, especially pressure, tries to 'fix' the dent and bring the shell to a perfect condition. In addition, from Fig. 4, the effect of non-proportional dent dimensions can be observed and conclude that a dent with a longer dimension perpendicular to a nominal stress is more dangerous. Thus, in dent assessment not only dent depth should be considered, but also length and width have to be taken into account. From Figs. 2-4, it's possible to conclude that internal pressure leads to bigger stress concentration factors with the same nominal stress level. 

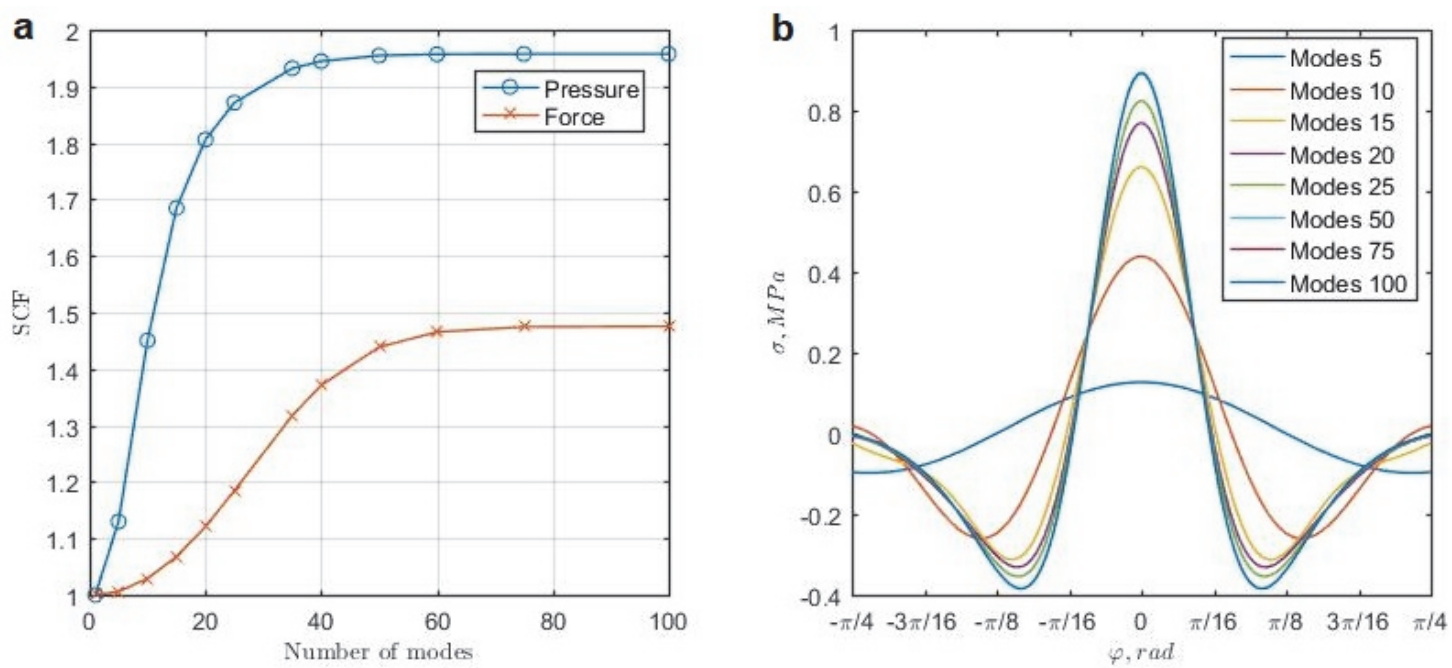

Figure 1: Convergence of results with respect to Fourier terms of expansion: a - stress concentrating factor for pressure and force loading; $\mathrm{b}$ - profiles of circumferential bending stress for pressure loading.
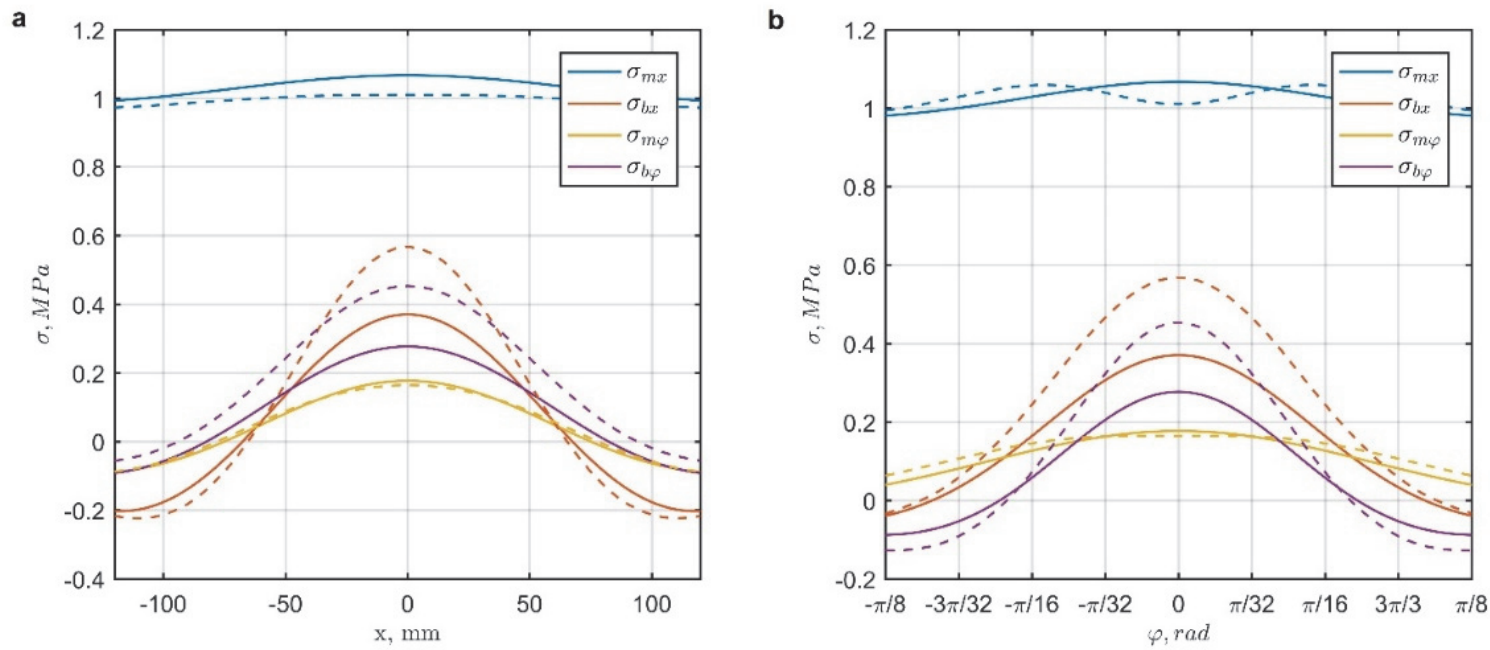

Figure 2: Comparison with FEM solution for axial force loading: $\mathrm{R}=400 \mathrm{~mm}, \mathrm{~h}=10 \mathrm{~mm}, \xi=0.5 \mathrm{~h}, \varphi_{0} \mathrm{R}=\chi_{0}=60 \mathrm{~mm}, \longrightarrow$ analytical solution; ....... FEM solution.
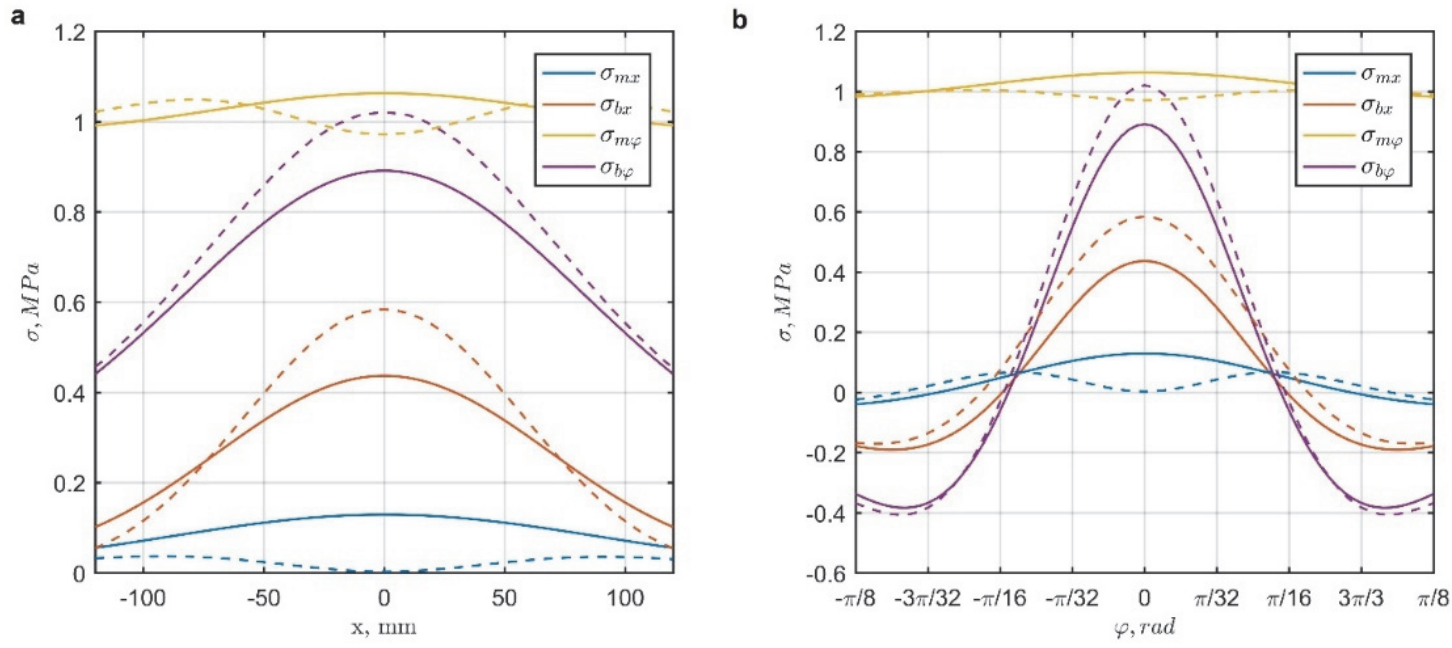

Figure 3: Comparison with FEM solution for internal pressure loading; $\mathrm{R}=400 \mathrm{~mm}, \mathrm{~h}=10 \mathrm{~mm}, \xi=0.5 \mathrm{~h}, \varphi_{0} \mathrm{R}=x_{0}=60 \mathrm{~mm}$, analytical solution; .......FEM solution. 

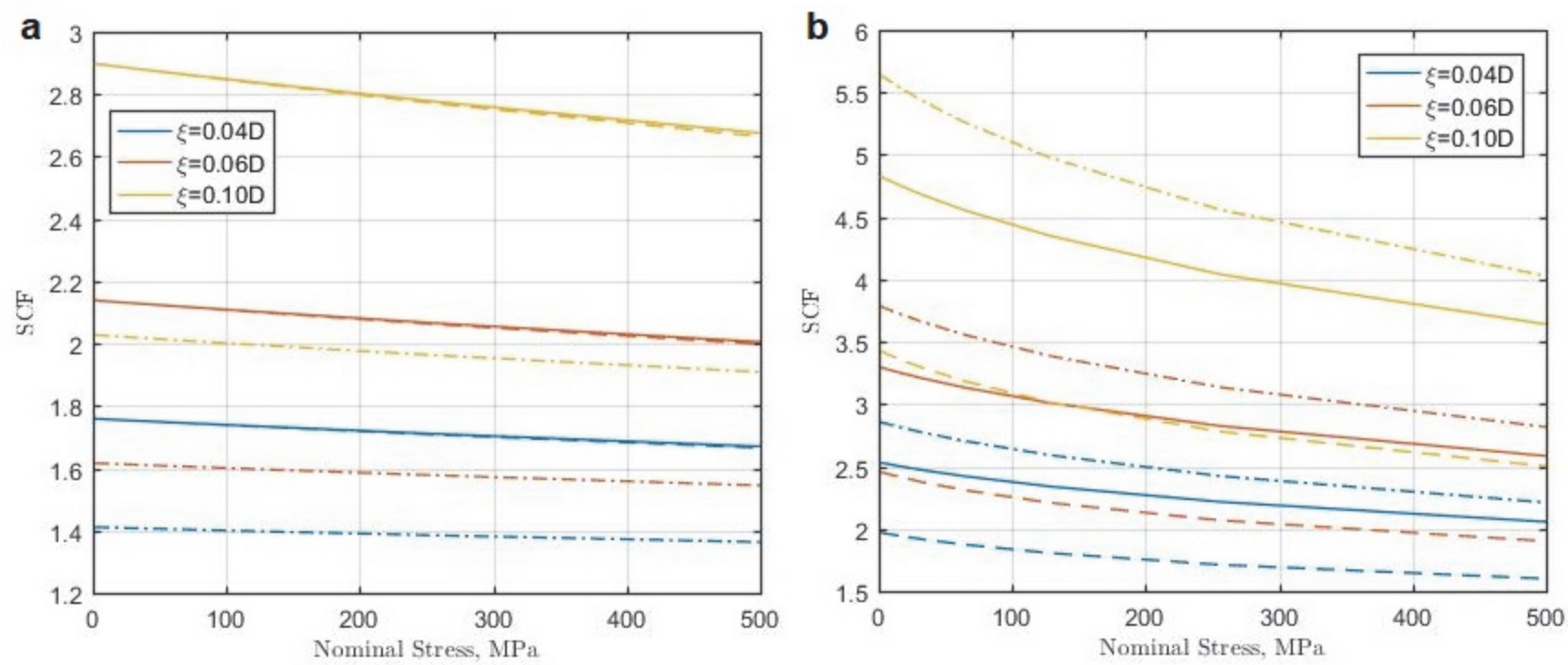

Figure 4: Effect of initial stress: a) Axial force loading, b) Internal pressure loading; $\mathrm{R}=400 \mathrm{~mm}, \mathrm{~h}=10 \mathrm{~mm}:-\varphi_{0} \mathrm{R}=\chi_{0}=60 \mathrm{~mm}$; $\varphi_{0} R=90 \mathrm{~mm}, x_{0}=60 \mathrm{~mm} ; \boldsymbol{-}-\varphi_{0} \mathrm{R}=60 \mathrm{~mm}, x_{0}=90 \mathrm{~mm}$.

In Fig. 5, the effect of shell radius to thickness ratio was analyzed and again it is possible to see the different behavior for internal pressure and axial force loading. It can be clearly seen from Fig. 5 that with increasing $\mathrm{R}$ to $\mathrm{h}$ ratio a higher SCF was obtained and the stress concentration can be up to 40 times. In oil and gas industries typical trunk pipelines have $\mathrm{R} / \mathrm{h}=15 \ldots 40$, so the results will be in the lower part of Fig. 5 and with the increasing of the nominal stress level SCF factor will decrease (see Fig. 4) and this effect will be more significant for flexible shells. Nevertheless, if using high strength steels for piping, the thickness can be reduced and must be aware of the increasing in SCF for dents in very flexible shells.
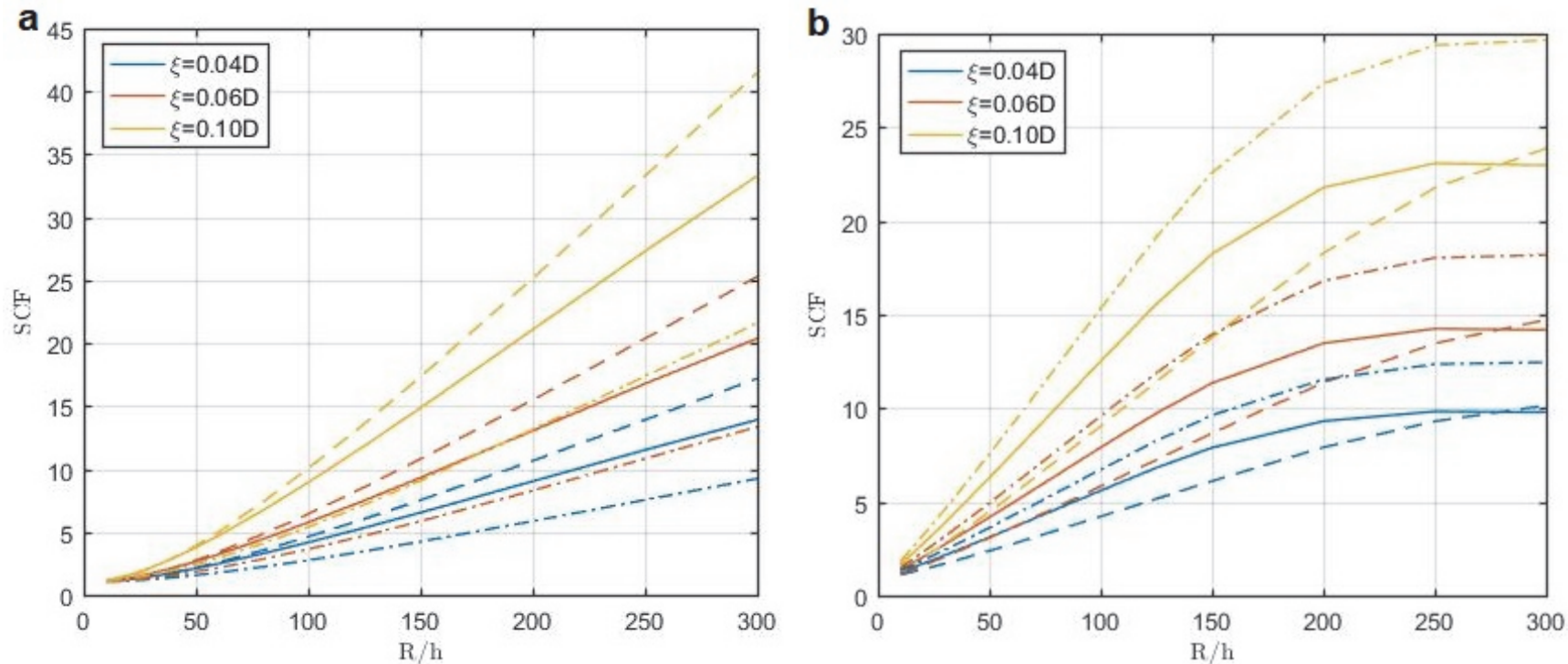

Figure 5: Effect of radius to thickness ration: a) Axial force loading, b) Internal pressure loading; $\sigma_{\text {nom }}=1 \mathrm{MPa}, \mathrm{h}=10 \mathrm{~mm}$ : $\varphi_{0} R=x_{0}=60 \mathrm{~mm} ; \ldots \ldots \ldots . \varphi_{0} R=90 \mathrm{~mm}, x_{0}=60 \mathrm{~mm} ; \ldots . \varphi_{0} \mathrm{R}=60 \mathrm{~mm}, x_{0}=90 \mathrm{~mm}$;

\section{CONCLUSIONS}

simple engineering approach was developed for analysis of stress-strain state of dented pipelines using equivalent load method. Using the harmonic imperfection model, an explicit analytical solution for a single dent was received and for a reliable accurate solution 50 terms in Fourie expansion must be used. Comparison of analytical stresses 
with the results of numerical simulation shows the effectiveness of the proposed procedures. However, equivalent load method underestimates the peak stress concentration value, but it predicts quite good the stress profile. The peak stress value can be adjusted by a simple semi-analytical correction.

Obtained semi-analytical solution allowed to conduct parametric studies for different dent shapes, radius to thickness ratio at different nominal stress level:

- for small dents with increasing depth the stresses concentration increases and for small dents, this growth was more significant

- the influence of pressure, for unproportional dent was more significant than in case of axial force loading;

- concentration due to the pressure load was more significant, and must be taken into account, especially for short dents, since it can exceed the nominal stress level by an excessive value;

- increasing in nominal stress level gives a reduction in stress concentration factor, and for pressure loading this decreasing was more significant;

- increasing in $\mathrm{R}$ to $\mathrm{h}$ ratio leads to increasing in stress concentration factor. For pressure and axial force loading this effect is different and should be accounted for very flexible pipelines.

The proposed semi-analytical solution is a powerful method for dents assessment, it is especially useful for parametric analysis, and it can be used to understand the factors that influence the pipeline safe operation.

\section{REFERENCES}

[1] EGIG. (2015). Gas Pipeline Incidents. 9th Report of the European Gas Pipeline Incident Data Group. Available at: https://www.egig.eu/reports/\$97/\$155.

[2] Kec, J. and Cerny, I. (2017). Stress-strain assessment of dents in wall of high pressure gas pipeline, Procedia Structural Integrity, 5, pp. 340-346. DOI: 10.1016/j.prostr.2017.07.180.

[3] Li, C., and Dang, C. (2017). Plastic damage analysis of oil and gas pipelines with unconstrained and constrained dents. Engineering Failure Analysis, 77, pp. 39-49. DOI: 10.1016/j.engfailanal.2017.02.009.

[4] Ying Wu, Y., Jiewen Xiao, J. and Peng Zhang, P. (2016). The analysis of damage degree of oil and gas pipeline with type II plain dent, Engineering Failure Analysis, 66, pp. 212-222. DOI: 10.1016/j.engfailanal.2016.04.004.

[5] Calladine, C. R. (1972). Structural Consequences of Small Imperfections in Elastic Thin Shells of Revolution, International Journal of Solids and Structures, 8(5), pp. 679-697. DOI: 10.1016/0020-7683(72)90036-4.

[6] Rinehart, A. J. and Keating, P. B. (2007). Stress Concentration Solution for a 2D Dent in an Internally Pressurized Cylinder, Journal of Engineering Mechanics, 133(7), pp. 792-800 DOI: 10.1061/(ASCE)0733-9399(2007)133:7(792).

[7] Godoy, L. A. (1996). Thin-Walled Structures with Structural Imperfections, New York, Pergamon. DOI: 10.1016/B978-0-08-042266-4.X5000-3.

[8] Godoy, L. A. (1993). On loads equivalent to Geometrical imperfections in shells, J. Eng. Mech, 119(1), pp. 186-190. DOI: 10.1061/(ASCE)0733-9399(1993)119:1(186).

[9] API 579-1/ASME FFS-1. (2016). Fitness-For-Service.

[10] Leissa, A. W. (1973). Vibration of Shells, NASA-SP-288. 